\title{
Existence of secondary flows in a reactive viscous fluid through a channel filled with a porous medium
}

\author{
Samuel O. Adesanya ${ }^{1,2 *}$, Michael B. Fakoya ${ }^{3}$, John A. Falade ${ }^{4}$, Ramoshweu S. Lebelo², \\ Dorcas M. Okewole ${ }^{1}$ \\ ${ }^{1}$ Department of Mathematical Sciences, College of Natural Sciences, Redeemer's University, Ede, \\ Nigeria \\ ${ }^{2}$ Department of Mathematics, Vaal University of Technology, Private Bag X021 Vanderbijlpark, \\ 1911, South Africa \\ ${ }^{3}$ Africa Centre for Sustainability Accounting and Management (ACSAM), School of Accountancy, \\ Faculty of Management and Law, University of Limpopo, Turfloop Campus, Polokwane, Limpopo \\ 0727, South Africa \\ ${ }^{4}$ Department of Physical Sciences, College of Natural Sciences, Redeemer's University, Ede,
} Nigeria

Email: adesanyas@run.edu.ng

\begin{abstract}
This paper investigates the existence of secondary flow superimposed on the reactive fluid a channel filled with porous materials. At maximum temperature, it is well-known that the energy equation has two solutions. For this purpose, the exact solution of the velocity profile is obtained and used to compute the multiple solutions arising from the nonlinear internal heat generation within the flow region. The effect of various flow parameters on the multiple solutions are presented graphically and discussed based on the physics of the fluid.
\end{abstract}

Keywords: Multiple Solutions, Secondary Flow, Porous Medium, Combustion, Adomian Decomposition Method.

\section{INTRODUCTION}

Flow and heat transfer processes in a saturated porous medium are frequently encountered in many thermal and technological processes in refineries, oil reservoirs, solar systems, nuclear reactors and many geophysical flows. In this regard, numerous researches have been conducted in this area of study, especially, on the flow of the strongly exothermic combustible fluid and accurate determination of safe working conditions to avoid thermal runaway. Of interest in this paper, is the multiplicity of solutions for the dimensionless nonlinear boundary value problem (BVP) arising from the momentum and energy equations given by Makinde [1] as follows:

$\frac{d^{2} u}{d y^{2}}-\beta^{2} u=-1 ; u(0)=0=u(1)$

$\frac{d^{2} \theta}{d y^{2}}+\lambda\left(e^{\frac{\theta}{1+\varepsilon \theta}}+\delta\left(\frac{d u}{d y}\right)^{2}+\delta \beta^{2} u^{2}\right)=0$

$\theta(0)=\theta(1)=0$ where $\lambda$ is the Frank-Kameneskii heat generation parameter, $\theta$ is the dimensionless temperature, $u$ is the dimensionless velocity, $\beta^{2}$ is the porous permeability parameter, $\delta$ is the viscous heating parameter and $\varepsilon$ is the activation energy parameter. Equations (1)-(2) models the hydrodynamically and thermally developed reactive fluid flow in horizontal parallel filled with porous material. In [1], the problem has been shown to have a solution by using perturbation techniques, In the case when $\varepsilon=0=\delta$, then (2) reduces to the well-known Bratu problem ([2]-[10]). The Bratu problem is the simplest combustion equation and has been used widely to test the efficacy of several analytical and numerical methods.

The existence of secondary flows in many heat transfer processes has been an age-long problem. For instance, Li and Liao [11], introduced a transformation to seek the multiple solutions to the strongly nonlinear Bratu problem. Moreover, Al-Refai [12], applied the idea of maximum principle to construct upper and lower solutions of a nonlinear elliptic equation that models the steady flow of non-isothermal permeable catalyst pellet with first order Arrhenius kinetics. Haberts and Gaudenzi [13] constructed upper and lower solutions to explain the existence and multiplicity of solutions 
for a nonlinear BVP. In [14], Naito and Tanaka implemented the Strum's comparison theorem together with shooting method to establish the existence of multiple solutions to a nonlinear BVP.

Moreover, from a cost accounting perspective, investments will go down the drain if the secondary flow superimposed on the primary flow is not checked. As a result, to enhance yields when working with reactive fluid in a channel filled with porous materials, there is need to minimize the secondary flows within the flow channel since it reduces the efficiency and performance of the entire engineering set up. For more on the material flow accounting, interested readers can see the comprehensive work in [15-19] for details.

In spite of several works done on reactive fluid flows, a survey of the literature showed clearly that existence of secondary flow in the class of reactive fluids had received little or no attention. Therefore, the specific objective of this paper is to investigate the existence of secondary flow inherent in a wide range of working reactive fluids especially channels filled up with porous materials. In the following section, we present the mathematical analysis.

\section{MATHEMATICAL ANALYSIS}

By using the method of an undetermined coefficient, equation (1) admits an exact solution of the form:

$u(y)=-\frac{e^{-y \beta}\left(-1+e^{y \beta}\right)\left(-e^{\beta}+e^{y \beta}\right)}{\left(1+e^{\beta}\right) \beta^{2}}$

As suggested in [1], the bifurcation analysis suggests that the problem will have two solution branches when $\lambda<\lambda_{c}$. The focus is on constructing the multiple solutions, so show the non-existence of a unique solution. To seek the solutions of the problem when $\lambda<\lambda_{c}$. Following [11], we set

$$
f(y)=e^{-\theta}
$$

introducing (4) in (2), we get

$$
\begin{aligned}
f^{\prime \prime}(y)= & \frac{\left[f^{\prime}(y)\right]^{2}}{f(y)} \\
& +\lambda+\delta \lambda f(y)\left(\beta^{2} u(y)^{2}+u^{\prime}(y)^{2}\right) \\
& f(0)=f(1)=1
\end{aligned}
$$

Evidently, the form of nonlinearity in the boundary value problem (5) is very tedious to solve. Therefore, we make a new transformation

$$
f(y)=1+S(y)
$$

Using (6) in (5), we obtain

$$
\left.\begin{array}{c}
S^{\prime \prime}(y)=-S(y) S^{\prime \prime}(y)+\left[S^{\prime}(y)\right]^{2}+\lambda(1+S(y)) \\
+\delta \lambda(1+S(y))^{2}\left(\beta^{2} u(y)^{2}+u^{\prime}(y)^{2}\right) ; \\
S(0)=S(1)=0
\end{array}\right\}
$$

The differential equation (7) is equivalent to the integral equation

$$
\begin{aligned}
S(y) & =\int_{0}^{y} S^{\prime}(0) d Y+\int_{0}^{y} \int_{0}^{y}\left[S^{\prime}(Y)\right]^{2}+\lambda(1+S(Y)) \\
& -S(Y) S^{\prime \prime}(Y) \\
+ & \delta \lambda(1+S(y))^{2}\left(\beta^{2} u(y)^{2}+u^{\prime}(y)^{2}\right) d Y d Y
\end{aligned}
$$

let $b=S^{\prime}(0)$, then the standard form of ADM [20] gives

$S(y)=\sum_{n=0}^{\infty} S_{n}(y)$

Substituting (9) in (8), we have

$$
\begin{aligned}
& \sum_{n=0}^{\infty} S_{n}(y)=b y+\int_{0}^{y} \int_{0}^{y}\left(\left[\sum_{n=0}^{\infty} S_{n}^{\prime}(Y)\right]^{2}\right) d Y d Y \\
& +\int_{0}^{y} \int_{0}^{y}\left(\lambda\left(1+\sum_{n=0}^{\infty} S_{n}(Y)\right)-\sum_{n=0}^{\infty} S_{n}(Y) \sum_{n=0}^{\infty} S_{n}^{\prime \prime}(Y)\right) d Y d Y \\
& +\int_{0}^{y} \int_{0}^{y}\left(\delta \lambda(1+S(y))^{2}\left(\beta^{2} u(y)^{2}+u^{\prime}(y)^{2}\right)\right) d Y d Y
\end{aligned}
$$

Let the nonlinear terms in (10) be

$$
\begin{aligned}
& A_{n}=\sum_{n=0}^{m} S_{n}^{\prime}(Y), B_{n}=\sum_{n=0}^{m} S_{n}(Y) \sum_{n=0}^{m} S_{n}^{\prime \prime}(Y), \\
& C_{n}=\left(1+\sum_{n=0}^{m} S(Y)\right)^{2}
\end{aligned}
$$

then the Adomian polynomials can be written as

$$
\begin{aligned}
& A_{0}=\left(S_{0}^{\prime}(Y)\right)^{2} \\
& A_{1}=2 S_{0}^{\prime}(Y) S_{1}^{\prime}(Y) \\
& A_{2}=2 S_{2}^{\prime}(Y) S_{0}^{\prime}(Y)+\left(S_{1}^{\prime}(Y)\right)^{2} \\
& \vdots \\
& B_{0}=S_{0}(Y) S_{0}^{\prime \prime}(Y) \\
& B_{1}=S_{0}(Y) S_{1}^{\prime \prime}(Y)+S_{1}(Y) S_{0}^{\prime \prime}(Y) \\
& B_{2}=S_{2}(Y) S_{0}^{\prime \prime}(Y)+S_{1}(Y) S_{1}^{\prime \prime}(Y)+S_{0}(Y) S_{2}^{\prime \prime}(Y)
\end{aligned}
$$

and

$$
\begin{aligned}
& C_{0}=\left(1+S_{0}(Y)\right)^{2} \\
& C_{1}=2\left(1+S_{0}(Y)\right) S_{1}(Y) \\
& C_{2}=S_{1}(Y)^{2}+2\left(1+S_{0}(Y)\right) S_{2}(Y)
\end{aligned}
$$

Given (12)-(14), we get the following iterative scheme 


$$
\begin{aligned}
S_{0}(y)= & b y+\int_{0}^{y} \int_{0}^{y} \lambda d Y d Y \\
S_{n+1}(y) & =\int_{0}^{y} \int_{0}^{y} A_{n}+\lambda S_{n}(Y)-B_{n} d Y d Y \\
& +\int_{0}^{y} \int_{0}^{y} \delta \lambda C_{n}\left(\beta^{2} u(y)^{2}+u^{\prime}(y)^{2}\right) d Y d Y
\end{aligned}
$$

Thus the partial sum

$$
S(y)=\sum_{n=0}^{j} S_{n}(y)
$$

gives the approximate solution to the problem, where $\mathrm{j}$ is the truncation point of the series. To compute the multiple solutions, the expression for the unknown constant is Taylor's series expanded up to the quadratic term (i.e., $S(1)=0$ ) in the form

$a_{0}+a_{1} b+a_{2} b^{2}=0$

returns two expressions to the unknown constant

$b_{1,2}=\frac{-a_{1} \pm \sqrt{a_{1}^{2}-4 a_{0} a_{2}}}{2 a_{0}}$

Hence by using the two constants in (18), we have two independent series solutions of the form

$\varphi_{1}(y)=\sum_{n=0}^{j} S_{n}(y), \varphi_{2}(y)=\sum_{n=0}^{j} S_{n}(y)$

Substituting (19) in (5), we get

$f_{1}(y)=1+\varphi_{1}(y), f_{2}(y)=1+\varphi_{2}(y)$

So that, the lower and upper solutions are:

$\theta_{L}(y)=-\log \left(1+\varphi_{1}(y)\right)$
$\theta_{U}(y)=-\log \left(1+\varphi_{2}(y)\right)$

Equations (1)-(21) are coded in Mathematica and the symbolic solutions are shown in Figures 1-7.

\section{RESULTS AND DISCUSSION}

Figures 1-3 represents the lower solution of the problem. Figure 1 captured the influence of the Frank-Kameneskii parameter on the temperature profile. From the graph, increasing values of the Frank-Kameneskii parameter elevates the temperature distribution of the fluid due to its exothermic nature. Also, Figure 2 represents the influence of viscous heating of the fluid on the thermal structure. The result shows that every increase in the viscous heating parameter has an increasing effect on the temperature distribution. This is due to continuous heat generation within the layers of the moving fluid. Figure 3 depicts the effect of porous permeability parameter on the temperature distribution. It is observed that rise in the porous permeability parameter $\beta^{2}$ implies a decrease in the porous permeability thus restricting the heat flow within the channel. This then resulted in reducing fluid temperature distribution within the channel. The upper and lower solutions are shown in Figure 4. As observed from the plot, the two solutions differ significantly and as such the solutions are not the same. However, Figure 5-7 represents the upper positive solution of the problem that satisfies the boundary conditions but its response to the variation of parameters does not obey any known physical law. Hence, the upper solution is a secondary heat and fluid flow which needed to be minimized for optimal performance of the system and improve profit margins.

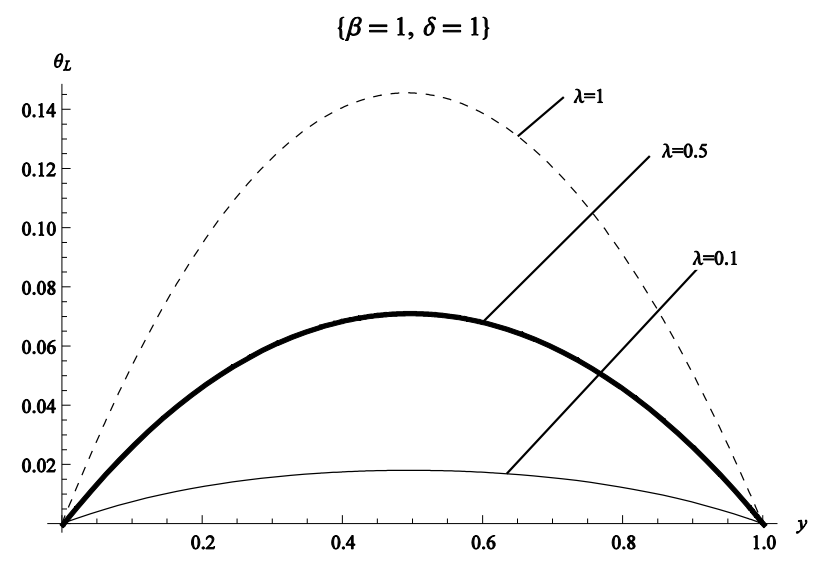

Figure 1. Frank-Kameneskii parameter effects on lower solution

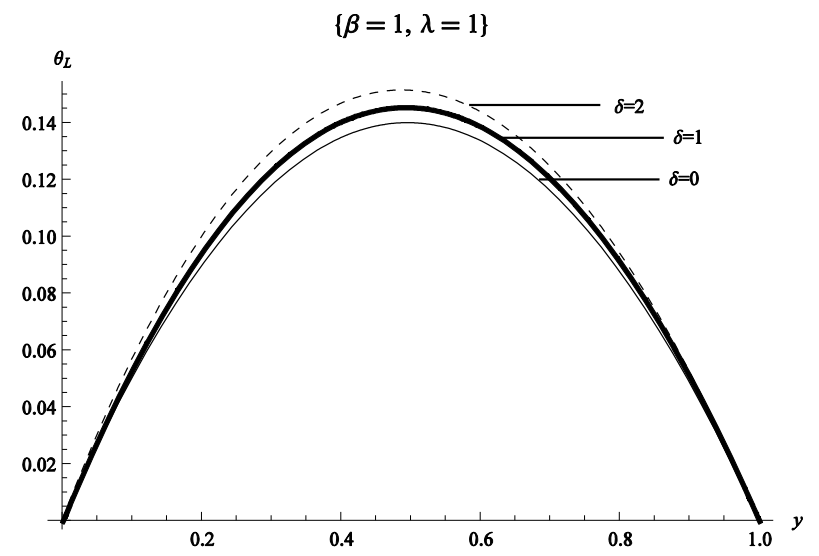

Figure 2. Viscous heating parameter effects on lower solution

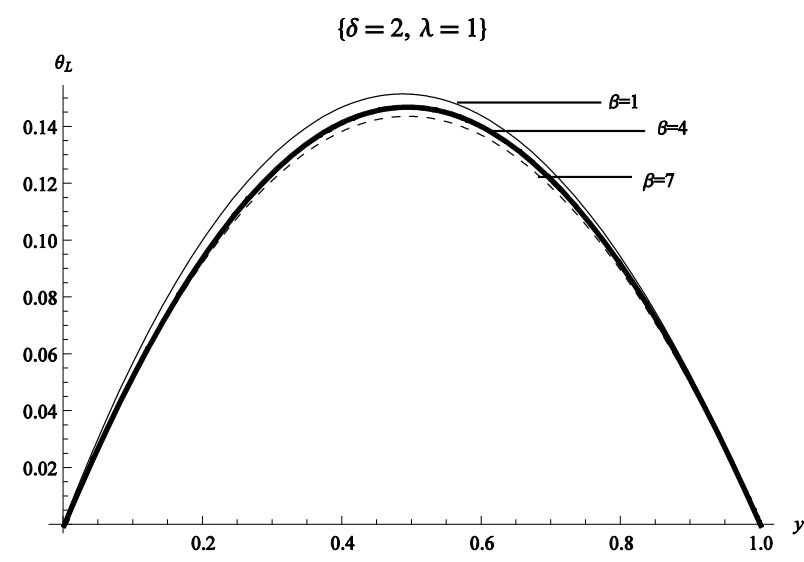

Figure 3. Porous permeability parameter effects on lower solution 


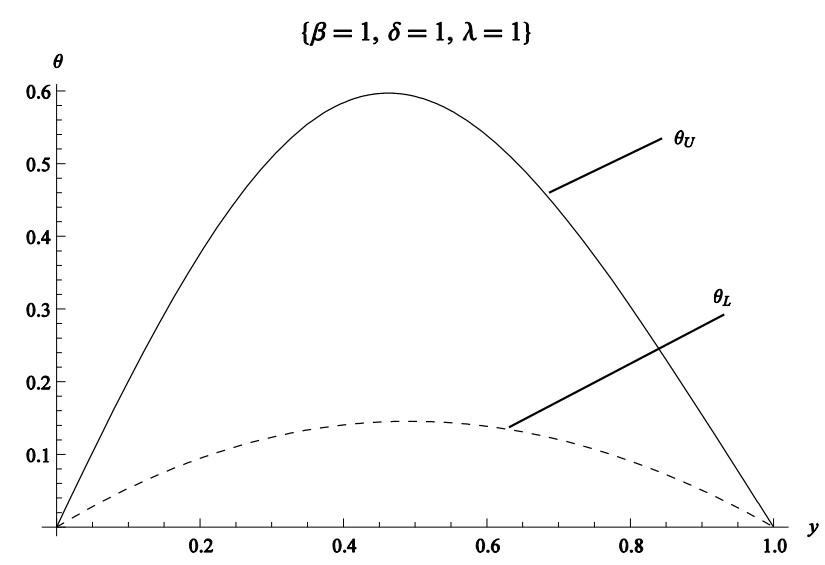

Figure 4. Multiple solutions

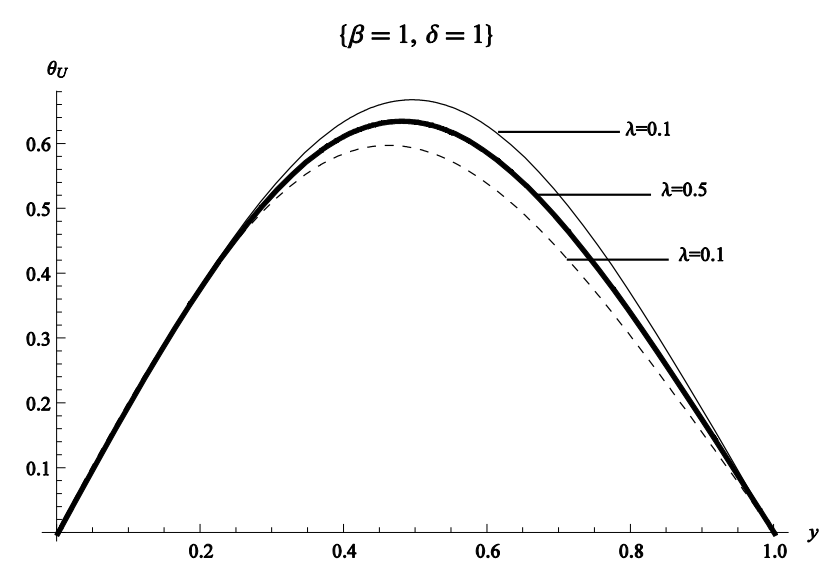

Figure 5. Effect of Frank-Kameneskii parameter on upper solution

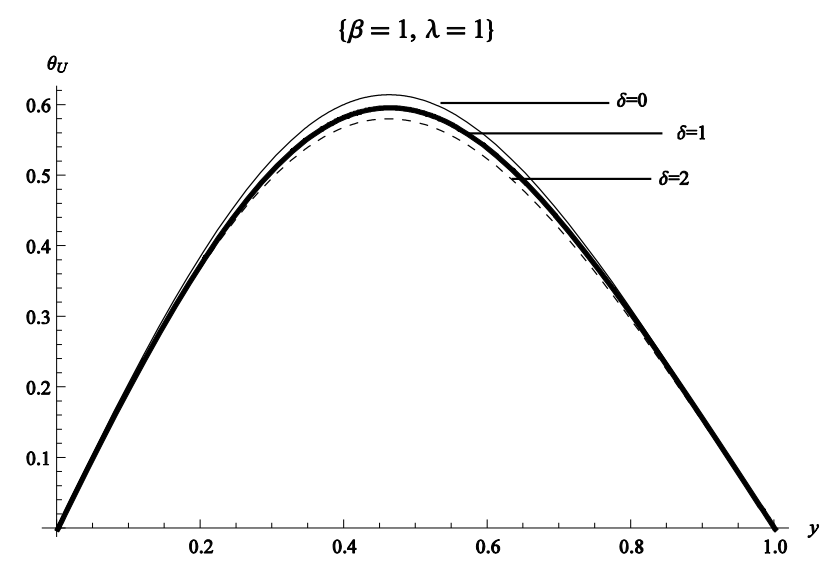

Figure 6. Effect of viscous heating parameter on upper solution

\section{CONCLUSION}

This paper reports the multiplicity of the solution to a nonlinear BVP that models reactive fluid flow in a channel saturated with a porous material. The dimensionless nonlinear BVP from the momentum and energy equations are solved and discussed appropriately. In our future work, the magnetic field effect on the non-Newtonian fluid flow based on [21-24] would be reported. Summarily, the major contributions from the present mathematical analysis are as follows:

i. the lower solution represents the primary heat flow, ii. the upper solution represents the secondary heat flow which needs to be controlled for optimal performance of the system.

\section{REFERENCES}

[1] Makinde_O.D., (2006) Thermal ignition in a reactive viscous flow through a channel filled with a porous medium, Journal of Heat Transfer, Vol. 128, No. 6, pp. 601-604. DOI: 10.1115/1.2188511

[2] Caglar H., Caglar N., Özer M., Valaristos A., Miliou A.N., Anagnostopoulos A.N. (2009). Dynamics of the solution of Bratu's equation, Nonlinear Analysis, Vol. 71, No. 12, pp. 672-678. DOI: 10.1016/J.NA.2008.11.091

[3] Syam M.I., Hamdan A. (2006). An efficient method for solving Bratu equations, Applied Mathematics and Computation, Vol. 176, pp. 704-713. DOI: 10.1016/J.AMC.2005.10.021

[4] Wang C.C. (2006). Use residual correction method to calculate the upper and lower solutions of initial value problem, Applied Mathematics and Computation, Vol. 181, pp. 29-39. DOI: 10.1016/J.AMC.2006.01.002

[5] Khuri S.A. (2004). A new approach to Bratu's problem, Applied Mathematics and Computation, Vol. 147, pp. 131-136. DOI: 10.1016/S0096-3003(02)00656-2

[6] Abbasbandy S., Hashemi M.S., Liu Chein-Shan (2011). The Lie-group shooting method for solving the Bratu equation, Commun Nonlinear Sci Numer Simulat, Vol. 16, pp. 4238-4249. DOI: 10.1016/J.CNSNS.2011.03.033

[7] Venkatesh S.G., Ayyaswamy S.K., Balachandar S.R. (2012). The Legendre wavelet method for solving initial value problems of Bratu-type, Computers and Mathematics with Applications, Vol. 63, pp. 12871295. DOI: 10.1016/J.CAMWA2011.12.069

[8] Aksoy Y, Pakdemirli M. (2010). New perturbationiteration solutions for Bratu-type equations, Computers and Mathematics with Applications, Vol. 59, pp. 28022808. DOI: 10.1016/J.CAMWA.2010.01.050

[9] Abbasbandy S., Shivanian E. (2010). Prediction of multiplicity of solutions of nonlinear boundary value problems: novel application of homotopy analysis method, Commun Nonlinear Sci Numer Simulat, Vol. 15, pp. 3830-3846. DOI: 10.1016/J.CNSNS.2010.01.030

[10] Buckmaster J.D., Ludford G.S.S. (1982). Theory of Laminar Flames, Cambridge University Press.

[11] Li S., Liao S.J. (2005). An analytic approach to solve multiple solutions of a strongly nonlinear problem, Applied Mathematics and Computation, Vol. 169, pp. 854-865. DOI: 10.1016/J.AMC.2004.09.066

[12] Refai M. (2006). Bounds and critical values for a combustion problem, Journal of Computational and Applied Mathematics, Vol. 188, pp. 33-43. DOI: 10.1016/J.CAM.2005.03.050

[13] Haberts P., Gaudenzi M. (1999). Existence and multiplicity of positive solutions for boundary value problems of 2D order ODE, Topological Methods in Nonlinear Analysis Journal of the Juliusz Schauder Center, Vol. 14, pp. 131-150. DOI: 10.12775/TMNA.1999.024

[14] Naito Y., Tanaka S. (2004). On the existence of multiple solutions of the boundary value problem for nonlinear second-order differential equations, 
Nonlinear Analysis, Vol. 56, pp. 919-935. DOI: 10.1016/J.NA.2003.10.020

[15] Fakoya M., van der Poll H. (2013). Integrating ERP and MFCA systems for improved waste-reduction decisions in a brewery in South Africa, Journal of Cleaner Production, Vol. 40, pp. 136-140. DOI: 10.1016/J.JCLEPRO.2012.09.013

[16] Kasemset C., Chernsupornchai J., Pala-ud W. (2015). Application of MFCA in waste reduction: case study on a small textile factory in Thailand, Journal of Cleaner Production, Vol. 108, pp. 1342-1351. DOI: 10.1016/J.JCLEPRO.2014.09.071

[17] Onishi Y., Kokubu K., Nakajima, M. (2008). Implementing material flow cost accounting in a pharmaceutical company, S. Schaltegger, M. Bennett, M. Burritt and C. Jasch, eds., Environmental Management Accounting for Cleaner Production, Springer, Netherlands, pp. 395-409.

[18] Songkham A., Kasemset C. (2015). Application of MFCA and dynamic programming in operations improvement: a case study, Industrial Engineering, Management Science and Applications, Springer, Berlin Heidelberg, pp. 35-44.

[19] Sygulla R., Götze U., Bierer A. (2014). Material flow cost accounting: A tool for designing economically and ecologically sustainable production processes, Technology and Manufacturing Process Selection, Springer, London, pp. 105-130.
[20] Adesanya S.O. (2013). Linear stability analysis of a plane-Poiseuille hydromagnetic flow using Adomian decomposition method, U.P.B. Sci. Bull., Series A, Vol. 75, pp. 99-106.

[21] Ireka I.E., T. Chinyoka (2013). Non-isothermal flow of a Johnson-Segalman liquid in a lubricated pipe with wall slip, Journal of Non-Newtonian Fluid Mechanics, Vol. 192, pp. 20-28. DOI: 10.1016/J.JNNFM.2012.11.001

[22] Rashad A.M., Rashidi M.M., Lorenzini G., Ahmed S.E., Aly A.M. (2017). Magnetic field and internal heat generation effects on the free convection in a rectangular cavity filled with a porous medium saturated with $\mathrm{Cu}-$ Water nanofluid, International Journal of Heat and Mass Transfer, Vol. 104, pp. 878-889.

DOI: 10.1016/j.ijheatmasstransfer.2016.08.025

[23] Singh J.K., Rohidas P., Joshi N., Begum S.G. (2017) Influence of Hall and ion-slip currents on unsteady MHD free convective flow of a rotating fluid past an oscillating vertical plate, International Journal of Heat and Technology, Vol. 35, No. 1, pp. 37-52. DOI: 10.18280/IJHT.350106.

[24] Rajput G.R., Krishnaprasad J.S.V.R., Timol M.G. (2016). Group theoretic technique for MHD forced convection laminar boundary layer flow of nanofluid over a moving surface, International Journal of Heat and Technology, Vol. 34, No. 1, pp. 1-6. DOI: 10.18280/IJHT.340101. 